\title{
Síndrome de Cushing iatrogênica em pacientes com HIV: análise da interação farmacológica entre esteróides e ritonavir
}

\author{
Iatrogenic Cushing syndrome in HIV patients: analysis of the pharmacological interaction between \\ steroids and ritonavir \\ Síndrome de Cushing iatrogénico en pacientes con VIH: análisis de la interacción farmacológica \\ entre esteroides y ritonavir
}

Ana Carolina Sardo de Oliveira Pinheiro ORCID: https://orcid.org/0000-0002-2405-8114 Universidade Federal do Pará, Brasil E-mail: sardcarolina@gmail.com

Beatriz Sayuri Vieira Ishigaki ORCID: https://orcid.org/0000-0001-6346-6025 Universidade do Estado do Pará, Brasil E-mail: beatrizishigaki00@gmail.com

Mariana Cristina Santos Andrade ORCID: https://orcid.org/0000-0002-9266-7800 Universidade Federal do Pará, Brasil

E-mail: marianaandrade192019@gmail.com

Arthur Cesar Batista Rodrigues ORCID: https://orcid.org/0000-0002-2659-3786 Universidade Federal do Pará, Brasil E-mail: arthur14032@gmail.com

Matheus Levy da Costa Freitas ORCID: https://orcid.org/0000-0002-9963-8842 Centro Universitário do Estado do Pará, Brasil E-mail: matheuslcfreitas@gmail.com

Gabriel de Sá Sastre

ORCID: https://orcid.org/0000-0001-9628-640X Universidade do Estado do Pará, Brasil E-mail: sastregabriell@gmail.com

Silas José Guimarães Pantoja Cardoso ORCID: https://orcid.org/0000-0002-1628-4429

Universidade do Estado do Pará, Brasil E-mail: silascardoso1103@hotmail.com

Bárbara Maria Paiva Corrêa ORCID: https://orcid.org/0000-0003-0484-9127 Universidade Federal do Pará, Brasil E-mail: baby11.04@hotmail.com

Jhonatan Lucas Ferreira Borges ORCID: https://orcid.org/0000-0002-3767-8283

Universidade do Estado do Pará, Brasil E-mail: jhonantanborges27@gmail.com

André Luiz Cardoso Cardoso ORCID: https://orcid.org/0000-0002-3740-4004 Universidade do Estado do Pará, Brasil

E-mail: andre.luiz.academico@gmail.com Thiago Santos Hirose ORCID: https://orcid.org/0000-0001-8654-0590 Centro Universitário Estácio, Brasi E-mail: thiago.hirose@gmail.com

\begin{abstract}
Resumo
Introdução: A Aids é uma doença que se caracteriza pela deficiência do sistema imunológico e que, no passado, trouxe para os seus portadores grande estigma social e baixa expectativa de vida. Com o aumento da expectativa de vida, surgiram novas questões de saúde dentre essa população relacionadas tanto ao envelhecimento quanto ao quadro de vulnerabilidade do HIV, o que resultou na complexidade inerente ao manejo farmacológico de doenças concomitante à terapia antirretroviral e seus riscos iatrogênicos inadvertidos, destacando-se assim a interação entre o
\end{abstract}


Ritonavir. Método: Foi realizada uma revisão de literatura nas bases de dados on-line da Biblioteca Virtual em Saúde (BVS), com a utilização dos descritores: "Iatrogenic Cushing's syndrome" AND "HIV" de 2011 a 2021. Resultados e discussão: 24 artigos foram encontrados, 2 eram revisões de literatura com abordagem sobre a relação entre o HIV e a síndrome de Cushing iatrogênica e 22 correspondiam a relatos de caso que tinham por base pacientes portadores de HIV em associação a doenças crônicas desenvolvidas pela idade, com destaque para as afecções osteomusculares, respiratórias e reumatológicas. Em função dessa combinação, a polifarmácia era aplicada, sendo o uso de corticoides e a terapia antirretroviral com o Ritonavir o fator farmacológico convergente entre todos os casos, bem como a principal manifestação clínica: A Síndrome de Cushing. Conclusão: Notou-se que o uso de glicocorticóides exógenos, como Triancinolona, Fluticasona e a Budesonida, que interagem com o Ritonavir, em pacientes que fazem uso de terapia antirretroviral do HIV pode induzir quadros clínicos da síndrome de Cushing.

Palavras-chave: Endocrinologia; HIV; Síndrome de Cushing.

\begin{abstract}
Introduction: Aids is a disease characterized by a deficiency of the immune system and which, in the past, brought great social stigma and low life expectancy to its patients. Associated with the increase in life expectancy, new health issues emerged among this population related to both aging and the vulnerability of HIV, which resulted in the inherent complexity of the pharmacological management of these diseases concomitant with antiretroviral therapy and their inadvertent iatrogenic risks, thus highlighting the interaction between Ritonavir. Method: A literature review was carried out in the online databases of the Virtual Health Library (VHL), using the descriptors: "Iatrogenic Cushing's syndrome" AND "HIV" from 2011 to 2021. Results and discussion: 24 articles found, 2 were literature reviews with an approach to the relationship between HIV and iatrogenic Cushing's syndrome and 22 corresponded to case reports that had based on patients with HIV in association with chronic diseases developed by age, with emphasis on musculoskeletal, respiratory and rheumatological disorders. Due to this combination, polypharmacy was applied, with the use of corticosteroids and antiretroviral therapy with Ritonavir the convergent pharmacological factor among all cases, as well as the main clinical manifestation: Cushing's Syndrome. Conclusion: It was noted that the use of exogenous glucocorticoids, like Triamcinolone, Fluticasone and Budesonide stand out, which interact with Ritonavir, in patients taking antiretroviral therapy for HIV can induce clinical presentations of Cushing's syndrome.
\end{abstract}

Keywords: Cushing's Syndrome; Endocrinology; HIV.

\title{
Resumen
}

Introducción: El sida es una enfermedad caracterizada por una deficiencia del sistema inmunológico y que, en el pasado, trajo un gran estigma social y una baja esperanza de vida a sus pacientes. Asociados con el aumento de la esperanza de vida, surgieron nuevos problemas de salud en esta población relacionados tanto con el envejecimiento como con la vulnerabilidad del VIH, que derivaron en la complejidad inherente del manejo farmacológico de estas enfermedades concomitantes con terapia antirretroviral y sus riesgos iatrogénicos inadvertidos, destacando así la interacción entre ritonavir. Método: Se realizó una revisión de la literatura en las bases de datos en línea de la Biblioteca Virtual en Salud (BVS), utilizando los descriptores: "Síndrome de Cushing iatrogénico" Y "VIH" de 2011 a 2021. Resultados y discusión: 24 artículos fueron encontrados, 2 fueron revisiones de literatura con un abordaje de la relación entre VIH y síndrome de Cushing iatrogénico y 22 correspondieron a reportes de casos que se habían basado en pacientes con VIH en asociación con enfermedades crónicas desarrolladas por edad, con énfasis sobre trastornos musculoesqueléticos, respiratorios y reumatológicos. Debido a esta combinación se aplicó polifarmacia, con el uso de corticoides y terapia antirretroviral con ritonavir el factor farmacológico convergente entre todos los casos, así como la principal manifestación clínica: el síndrome de Cushing. Conclusión: Se observó que el uso de glucocorticoides exógenos, como Triamcinolona, Fluticasona y Budesonida, que interactúan con ritonavir, en pacientes que toman terapia antirretroviral para el VIH puede inducir presentaciones clínicas del síndrome de Cushing.

Palabras clave: Endocrinología; Síndrome de Cushing; VIH.

\section{Introdução}

A Aids é uma doença que se caracteriza pela deficiência do sistema imunológico e que, no passado, trouxe para os seus portadores grande estigma social e baixa expectativa de vida (Fonseca, et al; 2020). Esse quadro tem sido modificado desde a década de 90 a partir do desenvolvimento de estudos e pesquisas voltadas ao tratamento com fármacos anti retrovirais, os quais elevaram a sobrevida desses pacientes (Araújo, Teva, Bermudez; 2014). Entretanto, associado ao aumento da expectativa de vida, surgiram novas questões de saúde dentre essa população relacionadas tanto ao envelhecimento quanto ao quadro de vulnerabilidade do HIV. Entre elas se destacam as doenças crônicas de afecção osteomuscular, pulmonar e reumatológica, o que resultou na complexidade inerente ao manejo farmacológico dessas doenças concomitante à terapia 
antirretroviral e seus riscos iatrogênicos inadvertidos, destacando-se assim a interação entre o Ritonavir - usado para potencializar os efeitos dos anti retrovirais - com os fármacos esteróides, úteis na terapia das doenças citadas. (Azevedo et al, 2015)

Dentre os principais efeitos adversos dessa interação farmacológica, a Síndrome de Cushing (SC) é uma das mais frequentes e graves (Joana, M; 2017). Essa é reconhecida na clínica pelos efeitos deletérios e estigmas visíveis nos tecidos periféricos devido à sobrecarga de glicocorticóides, sendo tal excesso resultado do acúmulo sistêmico de glicocorticóides endógenos ou exógenos, a depender de sua etiopatogenia (Benitez Arce; Aveiro; 2018). No caso dos pacientes com HIV, a etiologia da SC que os aflige, como já foi mencionada, é iatrogênica por ser resultado de interação farmacológica entre o Ritonavir e fármacos esteróides usados concomitantemente. (Colpitts et al, 2017)

O Ritonavir é um inibidor de protease e potente inibidor do citocromo P450 comumente associado a terapia em pacientes portadores de HIV por conta de seu efeito adjuvante a outros inibidores de protease ao aumentar a biodisponibilidade de substâncias primariamente eliminadas pela via metabólica CYP3A (referências). Assim, as drogas que são depuradas por essa via de metabolização, a exemplo dos macrolídeos e azólicos, além dos corticoesteróides, permanecem por tempo prolongado no organismo em virtude do bloqueio das vias de eliminação do citocromo P450. Destarte, a gênese da SC iatrogênica pode ser desencadeada pela interação entre o Ritonavir e os corticoesteróides, sobretudo quando são administradas drogas antiinflamatórias esteroidais em consonância. (Kedem et al, 2010)

Nesse sentido, essa pesquisa realizou um estudo sobre Relatos de Caso em que a administração de corticosteróides em pacientes portadores de HIV fazendo uso de Ritonavir levou ao desenvolvimento da SC. Esta análise foi realizada no intuito de identificar os fármacos esteróides que estão mais relacionados ao desenvolvimento da síndrome de Cushing nessa população e a justificativa farmacocinética para tal, já que o conhecimento acerca dessa interação é de suma importância no intuito de efetuar um manejo terapêutico seguro e cauteloso para pacientes adeptos a terapia antirretroviral com Ritonavir.

\section{Metodologia}

Foi realizada uma Revisão de Literatura Sistemática Integrativa na bases de dados on-line da Biblioteca Virtual em Saúde (BVS), tendo como data da pesquisa o dia 02 de março de 2021, com a utilização dos descritores: "Iatrogenic Cushing's syndrome" AND "HIV" de 2011 a 2021, o intervalo estudado se deve a escassez de informações nos últimos 5 anos acerca da temática, e com os filtros texto completo, ingles, português e espanhol. Sendo obtidos artigos no idioma português e inglês. Foram encontrados 32 artigos, e, após a leitura da base de revisão, foram selecionados 26 artigos, sendo 23 relatos de caso, 1 tese de mestrado e 2 revisões de literatura. Foram incluídos na pesquisa os artigos que continham informações sobre a interação farmacológica Ritonavir - corticosteróides e os seus efeitos colaterais para os pacientes e todos os artigos encontrados seguiram ao critério. Os critérios de inclusão foram: pacientes HIV positivos em uso de Ritonavir, artigos entre 2011 e 2021 , artigos em inglês, português e espanhol e os critérios de exclusão foram: pacientes com Síndrome de Cushing Iatrogênica que não fazem uso de Ritonavir, pacientes com lipodistrofia relacionada ao HIV e pacientes com problemas congênitos e intrínsecos relacionados ao metabolismo do cortisol. No mais, também foram incluídos no artigo mais 8 artigos, a partir dos descritores "Iatrogenic Cushing's syndrome" AND “Antiretroviral Therapy" e sem filtro de tempo, que continham temas para embasar a revisão de literatura.

\section{Resultados}

Após a aplicação dos filtros, foram encontrados 32 artigos, sendo destes selecionados 26 (tabela 1). Dentre os selecionados, 2 eram revisões de literatura com abordagem sobre a relação entre o HIV e a síndrome de Cushing iatrogênica e 
1 correspondia a uma tese de mestrado, enquanto que 23 correspondiam a relatos de caso que tinham por base pacientes portadores de HIV em associação a doenças crônicas desenvolvidas pela idade.

Os principais fármacos relacionados ao surgimento de Cushing Iatrogênico nos pacientes que fazem uso de Ritonavir foram: triancinolona, fluticasona e budesonida, sendo as apresentações injetáveis e inalatórias predominantes. Quanto ao tempo de uso do corticóide, foi observado que pacientes portadores de condições alérgicas crônicas levaram mais tempo para desenvolver alterações de cortisol em virtude da dosagem do esteróide ser menor, embora haja maior frequência de uso, enquanto que as injeções, sobretudo intra-articulares e epidurais, demonstraram ter efeito mais imediato produzindo Cushing Iatrogênico dentro de um intervalo de 2 semanas a 1 mês após o uso quando associada ao Ritonavir.

Tabela 1. Artigos selecionados para compor a revisão de literatura.

\begin{tabular}{|c|c|c|c|c|c|c|c|}
\hline Título & Ano & Autores & $\begin{array}{l}\text { Tipo de } \\
\text { estudo }\end{array}$ & Corticóide & Dose & $\begin{array}{l}\text { Tempo } \\
\text { de uso }\end{array}$ & Indicação \\
\hline $\begin{array}{c}\text { Ritonavir and epidural triamcinolone as a cause of } \\
\text { iatrogenic Cushing's syndrome }\end{array}$ & 2012 & $\begin{array}{l}\text { Nicole E. Albert, } \\
\text { Saifullah Kazi , Jerome } \\
\text { Santoro e Rebecca } \\
\text { Dougherty }\end{array}$ & $\begin{array}{l}\text { Relato de } \\
\quad \text { caso }\end{array}$ & Triancinolona & Não informado & 4 semanas & Estenose espinhal \\
\hline $\begin{array}{l}\text { Iatrogenic Cushing's syndrome and secondary } \\
\text { adrenal insufficiency after a single intra-articular } \\
\text { administration of Triamcinolone Acetonide in } \\
\text { HIV-infected patients treated with Ritonavir }\end{array}$ & 2008 & $\begin{array}{l}\text { JC Yombi , D. Maiter, } \\
\text { L. Belkhir, A. Nzeusseu } \\
\quad \text { e B. Vandercam }\end{array}$ & $\begin{array}{l}\text { Relato de } \\
\quad \text { caso }\end{array}$ & Triancinolona & $\begin{array}{l}40 \text { mg intra-articular } \\
\text { dose única }\end{array}$ & dose única & $\begin{array}{c}\text { Artrose } \\
\text { femoropatelar } \\
\text { externa, artrose da } \\
\text { coluna cervical e } \\
\text { periartrite } \\
\text { escapulo-umeral }\end{array}$ \\
\hline $\begin{array}{c}\text { Iatrogenic Cushing's syndrome and osteoporosis } \\
\text { due to an interaction between Fluticasone and } \\
\text { Ritonavir }\end{array}$ & 2015 & $\begin{array}{l}\text { Luísa azevedo, Hugo } \\
\text { Pêgo, Teresa Souto } \\
\text { Moura e Isabel Germano }\end{array}$ & $\begin{array}{l}\text { Relato de } \\
\quad \text { caso }\end{array}$ & Fluticasona & $\begin{array}{c}\text { Fluticasona } 250 \mathrm{mg} \\
\text { duas vezes ao dia } \\
\text { inalatória e } \\
\text { Fluticasona } \\
\text { intranasal } 110 \mathrm{mg} \\
\text { uma vez ao dia. }\end{array}$ & 5 anos & $\begin{array}{l}\text { Asma e rinite } \\
\text { alérgica }\end{array}$ \\
\hline $\begin{array}{c}\text { Fluticasone furoate induced iatrogenic Cushing } \\
\text { syndrome in a pediatric patient receiving anti- } \\
\text { retroviral therapy }\end{array}$ & 2017 & $\begin{array}{l}\text { SAA van den Berg, NE } \\
\text { Van 't Veer, JMA } \\
\text { Emmen, RHT van Beek }\end{array}$ & $\begin{array}{l}\text { Relato de } \\
\quad \text { caso }\end{array}$ & Fluticasona & $\begin{array}{c}27,5 \mathrm{mg} \text { em cada } \\
\text { narina duas vezes ao } \\
\text { dia }\end{array}$ & 2 meses & Rinite alérgica \\
\hline $\begin{array}{l}\text { Iatrogenic Cushing's Syndrome in patients } \\
\text { receiving inhaled Budesonide and Itraconazole or } \\
\text { Ritonavir: two cases and literature review }\end{array}$ & 2013 & $\begin{array}{l}\text { Marie-Christine Blondin, } \\
\text { Hugues Beauregard e } \\
\text { Omar Serri }\end{array}$ & $\begin{array}{l}\text { Relato de } \\
\text { caso e } \\
\text { revisão de } \\
\text { literatura }\end{array}$ & Budesonida & $\begin{array}{l}800 \mathrm{mg} \text { por dia } \\
\text { inalatória }\end{array}$ & $\begin{array}{l}\text { não } \\
\text { informado }\end{array}$ & Asma \\
\hline $\begin{array}{l}\text { Iatrogenic Cushing Syndrome in a 47-Year-Old } \\
\text { HIV-Positive Woman on Ritonavir and Inhaled } \\
\text { Budesonide }\end{array}$ & 2017 & $\begin{array}{l}\text { Lily Colpitts, Thomas B } \\
\text { Murray, Sami G. Tahhan } \\
\text { e Jody P. Boggs }\end{array}$ & $\begin{array}{l}\text { Relato de } \\
\quad \text { caso }\end{array}$ & Budesonida & $\begin{array}{l}160 \mathrm{mg} \text { por dia } \\
\text { inalatório }\end{array}$ & 6 meses & $\begin{array}{l}\text { Doença pulmonar } \\
\text { obstrutiva crônica }\end{array}$ \\
\hline $\begin{array}{c}\text { Acute development of Cushing's Syndrome in an } \\
\text { HIV-infected child on Atazanavir/Ritonavir-based } \\
\text { antiretroviral therapy }\end{array}$ & 2017 & $\begin{array}{c}\text { Gueorgui Dubrocq, } \\
\text { Andrea Estrada, } \\
\text { Shannon Kelly e Natella } \\
\text { Rakhmanina }\end{array}$ & $\begin{array}{l}\text { Relato de } \\
\quad \text { caso }\end{array}$ & Triancinolona & $\begin{array}{l}40 \text { mg intra-articular } \\
\text { dose única }\end{array}$ & Dose única & Artrite de joelho \\
\hline $\begin{array}{l}\text { Iatrogenic Cushing's syndrome and adrenal } \\
\text { insufficiency during concomitant therapy with } \\
\text { Ritonavir and Fluticasone }\end{array}$ & 2015 & $\begin{array}{l}\text { Narendranath Epperla e } \\
\text { Fergus McKiernan }\end{array}$ & $\begin{array}{l}\text { Relato de } \\
\quad \text { caso }\end{array}$ & Fluticasona & $\begin{array}{l}250 \mathrm{mcg} \text { inalatório } \\
\text { duas vezes ao dia }\end{array}$ & 3 anos & $\begin{array}{l}\text { Doença pulmonar } \\
\text { obstrutiva crônica }\end{array}$ \\
\hline $\begin{array}{l}\text { Pulmonary embolism and iatrogenic Cushing's } \\
\text { syndrome after co-administration of injected- } \\
\text { triamcinolone and ritonavir }\end{array}$ & 2013 & $\begin{array}{l}\text { Gregor John, Diana } \\
\text { Ollo, Patrick Meyer, } \\
\text { Caroline F Samer e } \\
\text { Alexandra Calmy }\end{array}$ & $\begin{array}{l}\text { Relato de } \\
\text { caso }\end{array}$ & Triancinolona & $\begin{array}{c}80 \text { mg intra-articular } \\
\text { dose única }\end{array}$ & Dose única & $\begin{array}{l}\text { Epicondilite } \\
\text { bilateral }\end{array}$ \\
\hline $\begin{array}{l}\text { Iatrogenic Cushing's Syndrome and multifocal } \\
\text { osteonecrosis caused by the interaction between } \\
\text { inhaled Fluticasone and Ritonavir }\end{array}$ & 2020 & $\begin{array}{l}\text { Joana Figueiredo, } \\
\text { Margarida Serrado, } \\
\text { Nikita Khmelinskii e } \\
\text { Sônia do Vale }\end{array}$ & $\begin{array}{l}\text { Relato de } \\
\text { caso }\end{array}$ & Fluticasona & $\begin{array}{l}250 \text { mcg inalatória } \\
\text { duas vezes ao dia }\end{array}$ & 5 anos & $\begin{array}{l}\text { Doença pulmonar } \\
\text { obstrutiva crônica }\end{array}$ \\
\hline
\end{tabular}




\begin{tabular}{|c|c|c|c|c|c|c|c|}
\hline $\begin{array}{l}\text { Iatrogenic Cushing syndrome after epidural } \\
\text { steroid injections for lumbar radiculopathy in an } \\
\text { HIV-infected patient treated with ritonavir: a case } \\
\text { report highlighting drug interactions for spine } \\
\text { interventionalists }\end{array}$ & 2012 & $\begin{array}{l}\text { Matthew J. Grierson e } \\
\text { Mark A. Harrast }\end{array}$ & $\begin{array}{l}\text { Relato de } \\
\text { caso }\end{array}$ & & & & $\begin{array}{l}\text { Radiculopatia } \\
\text { lombar }\end{array}$ \\
\hline $\begin{array}{l}\text { Iatrogenic Cushing syndrome after a single } \\
\text { intramuscular corticosteroid injection and } \\
\text { concomitant protease inhibitor therapy }\end{array}$ & 2011 & $\begin{array}{l}\text { Danielle Levine, Sonia } \\
\text { Ananthakrishnan, Amit } \\
\text { Garg }\end{array}$ & $\begin{array}{l}\text { Relato de } \\
\text { caso }\end{array}$ & Triancinolona & $\begin{array}{l}60 \text { mg dose única } \\
\text { intramuscular }\end{array}$ & Dose única & Dermatite \\
\hline $\begin{array}{l}\text { Iatrogenic cushing syndrome secondary to } \\
\text { ritonavir-epidural triamcinolone interaction: an } \\
\text { illustrative case and review. }\end{array}$ & 2014 & $\begin{array}{c}\text { Sapna Sadarangani, } \\
\text { Melody L. Berg, } \\
\text { William Mauck e Stacey } \\
\text { Rizza }\end{array}$ & $\begin{array}{l}\text { Relato de } \\
\text { caso e } \\
\text { revisão de } \\
\text { literatura }\end{array}$ & Triancinolona & $\begin{array}{l}\text { injetável } 40 \text { mg na } \\
\text { região cervical e } \\
20 \text { mg de injetável } \\
\text { na região da bursa } \\
\text { trocantérica }\end{array}$ & Dose única & Dor crônica \\
\hline $\begin{array}{c}\text { Iatrogenic Cushing's Syndrome Following Intra- } \\
\text { Articular Triamcinolone Injection in an HIV- } \\
\text { Infected Patient on Cobicistat Presenting as a } \\
\text { Pulmonary Embolism: Case Report and } \\
\text { Literature Review }\end{array}$ & 2020 & $\begin{array}{l}\text { Marjan Alidoost, } \\
\text { Gabriella A Conte, } \\
\text { Khushboo Agarwal, } \\
\text { Michael P Carson, } \\
\text { Danielle Lann e Diane } \\
\text { Marchesani }\end{array}$ & $\begin{array}{l}\text { Relato de } \\
\text { caso e } \\
\text { revisão de } \\
\text { literatura }\end{array}$ & $\begin{array}{l}\text { Triancinolona } \\
\text { e Fluticasona }\end{array}$ & Não informado. & 1 mês & $\begin{array}{l}\text { Cisto de Baker no } \\
\text { joelho e congestão } \\
\text { nasal }\end{array}$ \\
\hline $\begin{array}{l}\text { Iatrogenic cushing syndrome in an hiv-infected } \\
\text { patient secondary to concomitant therapy with } \\
\text { genvoya and epidural triamcinolone }\end{array}$ & 2020 & $\begin{array}{l}\text { Priyanka M Mathias e } \\
\text { Eric J Epstein }\end{array}$ & $\begin{array}{l}\text { Relato de } \\
\quad \text { caso }\end{array}$ & Triancinolona & $\begin{array}{l}80 \text { mg injeção } \\
\text { epidural }\end{array}$ & 18 meses & $\begin{array}{l}\text { Dor crônica na } \\
\text { coluna }\end{array}$ \\
\hline $\begin{array}{c}\text { Preventing Cushing: Iatrogenic Cushing } \\
\text { Syndrome due to Ritonavir-Fluticasone } \\
\text { Interaction }\end{array}$ & 2017 & $\begin{array}{l}\text { Fasil Tiruneh, Ahmad } \\
\text { Awan, Abiot Didana e } \\
\text { Saumil Doshi }\end{array}$ & $\begin{array}{l}\text { Relato de } \\
\text { caso }\end{array}$ & Fluticasona & $\begin{array}{l}200 \text { mcg intranasal } \\
\text { duas vezes por dia }\end{array}$ & 15 anos & Asma \\
\hline $\begin{array}{c}\text { Avascular Necrosis of Both Hips From Iatrogenic } \\
\text { Cushing's Syndrome due to Coadministration of } \\
\text { Fluticasone and Ritonavir in an HIV-Infected } \\
\text { Patient }\end{array}$ & 2020 & $\begin{array}{c}\text { Ravi Kant, Mark D } \\
\text { Cromer, Rashmi } \\
\text { Chandra, Kashif Munir e } \\
\text { Vipin Verma }\end{array}$ & $\begin{array}{l}\text { Relato de } \\
\text { caso }\end{array}$ & Fluticasona & $\begin{array}{l}100 \text { mcg intranasal e } \\
\text { aerossol duas vezes } \\
\text { por dia }\end{array}$ & $\begin{array}{c}\text { Não } \\
\text { informa }\end{array}$ & Asma \\
\hline $\begin{array}{l}\text { Iatrogenic Cushing syndrome after intra-articular } \\
\text { triamcinolone in a patient receiving ritonavir- } \\
\text { boosted darunavir }\end{array}$ & 2013 & $\begin{array}{l}\text { Jill J Hall, Christine A. } \\
\text { Hughes, Michelle M } \\
\text { Foisy, Stan Houston e } \\
\text { Stephen Shafran }\end{array}$ & $\begin{array}{l}\text { Relato de } \\
\text { caso }\end{array}$ & Triancinolona & $\begin{array}{l}40 \text { mg injetável } \\
\text { intra-articular dose } \\
\text { única }\end{array}$ & Dose única & $\begin{array}{l}\text { Dor crônica no } \\
\text { ombro }\end{array}$ \\
\hline $\begin{array}{l}\text { Iatrogenic Cushing's syndrome after } \\
\text { triamcinolone plus ritonavir-boosted atazanavir }\end{array}$ & 2015 & $\begin{array}{l}\text { Bernadette Jakeman, } \\
\text { Jessica Conklin, } \\
\text { Matthew Bouchonville e } \\
\text { Karla Thornton }\end{array}$ & $\begin{array}{l}\text { Relato de } \\
\text { caso }\end{array}$ & Triancinolona & $\begin{array}{c}20 \text { mg duas injeções } \\
\text { intrabursais dose } \\
\text { única }\end{array}$ & Dose única & $\begin{array}{c}\text { Bursite } \\
\text { subescapular }\end{array}$ \\
\hline $\begin{array}{c}\text { Síndrome de Cushing iatrogénica por interacção } \\
\text { entre fluticasona inalada e ritonavir }\end{array}$ & 2017 & $\begin{array}{c}\text { Joana Margarida Neves } \\
\text { Figueiredo }\end{array}$ & $\begin{array}{l}\text { Tese de } \\
\text { mestrado }\end{array}$ & Fluticasona & $250 \mathrm{mcg}$ inalatória & 5 anos & $\begin{array}{l}\text { Doença pulmonar } \\
\text { obstrutiva crônica }\end{array}$ \\
\hline $\begin{array}{l}\text { Inhaled Fluticasone Causes Iatrogenic Cushing's } \\
\text { Syndrome in Patients Treated with Ritonavir }\end{array}$ & 2011 & $\begin{array}{l}\text { KEREN MAHLAB- } \\
\text { GURI, ILAN ASHER, } \\
\text { SERGE GRADSTEIN, } \\
\text { AMNON ZUNG, } \\
\text { SARA RADIAN-SADE, } \\
\text { DANIEL ELBIRT e } \\
\text { ZEV STHOEGER }\end{array}$ & $\begin{array}{l}\text { Relato de } \\
\text { caso }\end{array}$ & Fluticasona & $\begin{array}{l}50 \text { a } 200 \mathrm{mcg} \\
\text { inalatória duas vezes } \\
\text { ao dia }\end{array}$ & $\begin{array}{l}6 \text { meses a } 2 \\
\text { anos }\end{array}$ & Asma \\
\hline $\begin{array}{l}\text { Injecting epidural and intra-articular } \\
\text { triamcinolone in HIV-positive patients on } \\
\text { ritonavir: beware of iatrogenic Cushing's } \\
\text { syndrome }\end{array}$ & 2013 & $\begin{array}{l}\text { M. Maviki, P. Cowley e } \\
\text { H. Marmery }\end{array}$ & $\begin{array}{l}\text { Relato de } \\
\text { caso }\end{array}$ & Triancinolona & $\begin{array}{c}80 \mathrm{mg} \text { injetável dose } \\
\text { única }\end{array}$ & dose única & $\begin{array}{l}\text { Dor lombar e } \\
\text { bursite } \\
\text { subacromial }\end{array}$ \\
\hline $\begin{array}{l}\text { Clinical Course and Management of Iatrogenic } \\
\text { Cushing's Syndrome after Co-Administration of } \\
\text { Injected-Triamcinolone and Ritonavir: a } \\
\text { Systematic Review }\end{array}$ & 2013 & $\begin{array}{l}\text { Gregor John, Diana } \\
\text { Ollo, Patrick Meyer, } \\
\text { Markus Herold, Caroline } \\
\text { Flora Samer e Alexandra } \\
\text { Calmy }\end{array}$ & $\begin{array}{c}\text { Revisão de } \\
\text { literatura }\end{array}$ & Triancinolona & $\begin{array}{l}40 \text { a } 240 \mathrm{mg} \\
\text { injetável }\end{array}$ & $\begin{array}{l}\text { Não } \\
\text { informado }\end{array}$ & Dor crônica \\
\hline $\begin{array}{c}\text { Inhaled corticosteroid use in HIV-positive } \\
\text { individuals taking protease inhibitors: a review of } \\
\text { pharmacokinetics, case reports and clinical } \\
\text { management }\end{array}$ & 2013 & $\begin{array}{l}\text { P Saberi, T } \\
\text { Phengrasamy, DP } \\
\text { Nguyen }\end{array}$ & $\begin{array}{c}\text { Revisão de } \\
\text { literatura }\end{array}$ & $\begin{array}{c}\text { Fluticasona e } \\
\text { budesonida }\end{array}$ & $\begin{array}{l}100 \text { a } 1200 \mathrm{mg} \\
\text { intranasal por dia }\end{array}$ & $\begin{array}{l}2 \text { a } 18 \\
\text { meses }\end{array}$ & $\begin{array}{l}\text { Asma, rinite e } \\
\text { DPOC }\end{array}$ \\
\hline
\end{tabular}




\begin{tabular}{|c|c|c|c|c|c|c|c|}
\hline $\begin{array}{c}\text { Triamcinolone and ritonavir leading to drug- } \\
\text { induced Cushing syndrome and adrenal } \\
\text { suppression: description of a new case and review } \\
\text { of the literature }\end{array}$ & 2013 & $\begin{array}{l}\text { C. Schwarze-Zander, D. } \\
\text { Klingmüller, J. Klümper, } \\
\text { C. P. Strassburg \& J. K. } \\
\text { Rockstroh }\end{array}$ & $\begin{array}{l}\text { Relato de } \\
\text { caso }\end{array}$ & Triancinolona & $\begin{array}{l}20 \text { mg injetável } \\
\text { perriradicular } \\
\text { semanalmente }\end{array}$ & 6 meses & $\begin{array}{l}\text { Estenose } \\
\text { foraminal lombar } \\
\text { bilateral }\end{array}$ \\
\hline $\begin{array}{l}\text { Cushing's Syndrome with Adrenal Suppression } \\
\text { Induced by Inhaled Budesonide Due to a } \\
\text { Ritonavir Drug Interaction in a Woman with HIV } \\
\text { Infection }\end{array}$ & 2012 & $\begin{array}{l}\text { K. Yoganathan, L. David } \\
\text {, C. Williams e K. Jones }\end{array}$ & $\begin{array}{l}\text { Relato de } \\
\text { caso }\end{array}$ & Budesonida & $\begin{array}{l}400 \text { mcg inalatória } \\
\text { duas vezes ao dia }\end{array}$ & $\begin{array}{l}1 \text { ano e } 3 \\
\text { meses }\end{array}$ & Asma \\
\hline
\end{tabular}

Fonte: Autores.

\section{Discussão}

A maior parte dos relatos de caso que tinham por base pacientes portadores de HIV que também eram portadores de doenças crônicas desenvolvidas pela idade, como afecções osteomusculares, respiratórias e reumatológicas. Em função dessa combinação, a polifarmácia era aplicada, sendo o uso de corticoides e a terapia antirretroviral com o Ritonavir o fator farmacológico convergente entre todos os casos, bem como a principal manifestação clínica: A Síndrome de Cushing. Tal efeito sindrômico presente nesse grupo, que em alguns casos era acompanhado por insuficiência adrenal secundária em virtude do bloqueio do eixo hipotálamo-hipófise-adrenal (HPA) pelo excesso de corticóide circulante, originava-se da interação medicamentosa entre Corticoides e o Ritonavir, cuja ação inibitória sobre enzimas hepáticas, especialmente sobre o conjunto CYP3A4, causa redução na metabolização dos fármacos esteróides e, consequentemente, seu acúmulo sistêmico e prolongamento no tempo de ação sobre os receptores. (Jakeman et al, 2015.).

De acordo com Epperla et al (2015) e Saberi et al (2013) como principais efeitos da combinação medicamentosa esteroide-Ritonavir, destacam-se a osteoporose, fácies cushingóide, adiposidade central, estrias púrpuras, fraqueza muscular e o ganho de peso. Essas manifestações são variáveis de acordo com o perfil de cada paciente, sendo as mais sutis o ganho de peso e a distribuição da gordura corporal e as mais severas a hipertensão, resistência insulínica, pré-diabetes e diabetes mellitus ao ponto de gerar a hiperglicemia hiperosmótica, síndrome metabólica, necrose avascular de quadril (Kant, et al., 2020), miopatia induzida por esteroide e reativação da herpes zoster, em virtude da imunossupressão. (John et al., 2013; Alidoost, et al., 2020; Kant, et al., 2020; Tiruneh, et al., 2017; Berg, et al., 2017; Guri, et al, 2011; Figueiredo, et al, 2020; Epperla \& Fergus, 2015; Yoganathan, et al, 2012; Colpitts, et al, 2017; Blondin, et al, 2013.).

Adicionalmente, outros estudos indicam como os principais achados relacionados ao uso indiscriminado de corticoesteróides-ritonavir, a presença de grandes efeitos adversos, refletidos no aumento da pressão arterial, aumento da glicemia, insuficiência renal e até mesmo o agravamento da supressão da resposta imune tanto inata quanto adquirida. Esse estudo também pontua que a dose e a duração da exposição determinam a toxicidade, a qual é traduzida pelo nível de supressão do eixo hipotálamo-hipófise-adrenal (Jakeman et al, 2015).

Nesse sentido, devido à presença de sinais e sintomas característicos, o diagnóstico da síndrome de Cushing é essencialmente clínico, embora seja confirmado a partir da baixa dosagem cortisol plasmática matinal, que revela a resposta anormal suprimida de ACTH e CRH. A clínica é evidenciada por presença de estria abdominal, pletora facial, obesidade central, acne, acantose nigricans e outros que configuram a síndrome cushingóide. (Dubrocq et al, 2017; Epperla et al, 2015)

É necessário, no entanto, distinguir a síndrome de Cushing e as síndromes de lipodistrofia relacionadas ao HIV-1. Embora a sintomatologia curse de forma semelhante à SC, com ganho de peso, adiposidade central, corcunda dorsocervical, resistência a insulina, osteropenia, dislipidemia, atrofia periférica e lipoacumulação central, incluindo hipertrofia mamária, a ausência de estrias abdominais favorece a síndrome da lipodistrofia associada à terapia antirretroviral, todavia é mandatória solicitar a dosagem de cortisol matinal a fim de firmar diagnóstico. (Mahlab-gur et al, 2011; Epperla \& Fergus, 2015; Schwarze-zander et al, 2013). 
Tão importante quanto estabelecer o diagnóstico sindrômico, que acarreta maior sofrimento a pacientes já enfraquecidos pelo HIV, é identificar a etiologia. Nos relatos de caso foi possível analisar quais esteróides exógenos estão mais associados ao desenvolvimento do Cushing iatrogênico, destacando-se a Fluticasona, Budesonida e Triancinolona, a justificativa farmacocinética para a interação desses com o Ritonavir, bem como as alternativas farmacológicas para os casos de supressão do eixo Hipotálamo-hipófise-Adrenal (HPA) (Maviki et al, 2013).

Dessa forma, foi observado que o uso da Fluticasona inalatória em associação com o Ritonavir foi evidenciado em 11 dos 23 Relatos de Caso analisados neste estudo, sendo utilizada com a finalidade de resolver as afecções pulmonares crônicas sofridas pelos pacientes (DPOC, Asma, Rinite). Embora a via de administração deste fármaco seja segura e eficaz, isto é, pouco associada a efeitos adversos sistêmicos entre os Glicocorticóides que apresentam interação com Ritonavir, é consenso que a Fluticasona é o esteróide exógeno com maior predisposição a desencadear tais eventos. (Saberi, et al, 2013; Epperla \& Fergus, 2015; Neto, et al, 2010).

Esses efeitos são resultado das características farmacodinâmicas e farmacocinéticas inerentes a Fluticasona, já que além de possuir alta lipofilicidade, também apresenta grande afinidade pelo receptor dos glicocorticóides, o que facilita a passagem pela membrana plasmática da célula alvo e potencializa a ativação dos receptor intracelulares e, consequentemente ação a nível gênico. Além disso, quando comparado a outros fármacos esteróides, este fármaco apresenta meia-vida prolongada (10 horas) e, principalmente, é em quase sua totalidade metabolizado pelo sistema enzimático CYP3A4. (Johann et al., 2013; Alidoos, et al., 2020; Kant, et al., 2020; Tiruneh, et al., 2017; Berg, et al., 2017; Saberi, et al, 2013; Guri, et al; 2011; Figueiredo, et al, 2020; Epperla \& Fergus, 2015; Azevedo, et al, 2015.).

Nesse sentido, o uso concomitante deste esteroide exógeno com o Ritonavir, que promove a inibição da enzima CYP3A4, resulta no aumento da área abaixo da curva concentração-tempo, em outras palavras, eleva a biodisponibilidade e prolonga o tempo de meia-vida da Fluticasona, o que acarreta o seu acúmulo sistêmico refletido no quadro característico da síndrome de Cushing (Epperla \& Fergus, 2015; Azevedo, et al, 2015.), a qual pode ser seguida por insuficiência adrenal secundária, caso seja feita a interrupção abrupta do uso do esteróide. Ademais as manifestações clínicas dessa síndrome são variáveis em quantidade e intensidade de sintomas, a depender da dose e do tempo de administração, sendo altas doses e tempo prolongado de terapia concomitante associados a potencialização dos efeitos sistêmicos e ao aumento do período de recuperação do eixo HPA (Epperla \& Fergus, 2015; Azevedo, et al, 2015; Neto, et al, 2010). Como foi observado nos relatos, a maioria dos pacientes apresentavam quadros crônicos de média a grave intensidade, o que demandava a prescrição de doses mais altas da Fluticasona por um tempo prolongado e resultava não apenas na Síndrome de Cushing, mas também em fragilidade óssea, efeitos psíquicos e metabólicos, com período de recuperação da função adrenal e do eixo HPA de em média 6 meses (Epperla \& Fergus, 2015; Gregor, et al., 2013).

Os artigos também destacam a existência de relatos nos quais a síndrome resultou do uso de Ritonavir com baixas doses de fluticasona, embora o quadro esteja mais relacionado às doses altas de corticóides. (Saberi, et al, 2013.). Por fim, é importante destacar que embora o FDA não recomenda o uso concomitante de fluticasona com o ritonavir (a não ser que o benefício supere os riscos) (Epperla \& Fergus, 2015; Colpitts, et al, 2017.) o número de relatos sugere relativa frequência de prescrição dessa medicação.

Outro fármaco que também gerou a síndrome de Cushing nos pacientes com HIV em terapia com o Ritonavir foi a Budesonida. Neste estudo, foram encontrados 3 relatos de caso a respeito dessa interação, demonstrando que embora esse esteroide seja uma das alternativas para a substituição da Fluticasona, também pode proporcionar efeitos adversos semelhantes (Yoganathan, et al, 2012; Colpitts, et al, 2017; Blondin, et al, 2013). Isso também se deve às propriedades farmacodinâmicas e farmacocinéticas da Budesonida, a qual embora apresente menor afinidade pelo receptor de glicocorticoide, alta inativação pelo metabolismo de primeira passagem, curta meia-vida (3h) e menor volume de distribuição, quando comparada a 
Fluticasona, possui naturalmente maior biodisponibilidade (32\%, enquanto a Fluticasona é de <1\%) e também sofre metabolização pelo sistema enzimático inibido pelo Ritonavir (Chong et al, 2010; Tashkin, et al, 2019; Yoganathan, et al, 2012; Colpitts, et al, 2017; Blondin, et al, 2013.), apresentando como resultado a potencialização do seu efeito sistêmico.

Além disso, embora a maioria dos relatos encontrados sejam sobre a interação com Ritonavir, um deles retrata a interação farmacológica com o Itraconazol em combinação com terapia antirretroviral associada ao Ritonavir e a Budesonida. Esse estudo, trouxe a perspectiva desse outro fármaco que também apresenta atividade inibitória na isoenzima CYP3A4, e que foi utilizado para o tratamento de pneumonia por agente fúngico, comum em pacientes com imunodeficiência. Nesse sentido, foi observado que o Itraconazol pode aumentar em 4.2 x e 1,6x a área abaixo da curva concentração-tempo e a concentração máxima de budesonida, respectivamente, também gerando aumento no tempo de meia-vida do fármaco em 1.6x (Blondin, et al, 2013.). Como consequência, a interação de fármacos de ação inibitória semelhante a CYP3A4 com a Budesonida pode gerar a síndrome de Cushing, mesmo em doses baixas, como foi relatado. Dessa maneira, para pacientes com HIV, a atenção deve ser redobrada quando em associação com os problemas crônicos também ocorrem infecções oportunistas fúngicas que são usualmente tratadas pelo Itraconazol.

Sob essa perspectiva, a busca por opções farmacológicas converge na literatura para 3 alternativas: trocar o ritonavir por um fármaco que não tenha ação sobre a CYP3A4, reduzir a dose do fármaco esteróide ou substituí-lo pela Hidrocortisona ou Beclometasona. Nos casos estudados, foi observado, predominantemente, a escolha pela terceira opção com bons resultados, embora demorados. Entre as duas opções farmacológicas apresentadas, a beclometasona obteve destaque, sendo sua farmacocinética e farmacodinâmica descritas como de significativa biodisponibilidade, afinidade ao receptor intracelular semelhante a Fluticasona, tempo de meia-vida intermediário e alta lipofilicidade (Chong Neto, et al, 2010.).

Essas características, por serem semelhantes a Fluticasona, sugerem que esse fármaco não seria uma boa opção para substituição. Contudo, a Beclometasona possui uma propriedade chave para justificar a sua escolha: A metabolização por outras esterases. Assim, embora essa substância também sofra a metabolização pelo CY3PA4, apresenta outras opções enzimáticas que impedem a acumulação tecidual do corticoide, impedindo a interferência farmacológica com o Ritonavir e suas consequências hormonais (Epperla \& Fergus, 2015; Blondin, et al, 2013.)

Ainda, outro fármaco destacado em 13 artigos entre os que foram revisados é a triancinolona acetonida, um corticóide sintético que é disponível na forma tópica, injetável e spray nasal. Sua aplicabilidade é relacionada a dermatites, alívio de afecções intra-articulares e atopias nasais e a metabolização é predominantemente hepática, o que justifica sua elevada interação com medicamentos bloqueadores da CYP3A4, como o ritonavir.

Dentre os relatos, a via de administração que mais causou efeitos e também a que mais fez usada foi a via injetável epidural para tratar afecções osteoarticulares, nesse caso, sabe-se que por essa via a triancinolona continua a ser absorvida pelos tecidos moles por 2-3 semanas após a dose, o que potencializa os efeitos adversos relacionados ao cushing (Maviki et al,2013).

Embora não hajam muitos relatos que apresentaram os efeitos de via de administração intramuscular, Levine e colaboradores (2011) demonstraram que essa via de administração é músculo/gordura dependente quando se trata da absorção, logo pode resultar em maior toxicidade a depender da massa gorda do paciente. Um estudo de revisão dos corticóides inalatórios demonstrou que, dentre o grupo, a triancinolona é um dos que apresentam menor lipofilicidade e, portanto, menos efeitos sistêmicos (Saberi et al., 2013). Todavia, sabe-se que tanto as vias injetáveis, quanto oral e tópica são capazes de provocar SC exógena na dependência da dosagem e tempo de uso.

Quanto à farmacocinética, em semelhança aos demais corticóides sintéticos, o tempo de meia vida da triancinolona é em torno de 3,1 horas, no entanto, o estudo de Sadarangani et al (2014) demonstrou que o tempo de meia vida aumenta cerca de 170 vezes quando associado ao ritonavir, fator que prejudica a melhora da paciente quanto aos efeitos sindrômicos, haja 
vista que o curso clínico dos pacientes relatados apresentou melhora entre 3 e 6 meses, a depender dos sintomas (Levine D et al, 2011). Schwarze-Zander e colaboradores (2013), demonstraram em seu estudo que uma única dose injetada de $40 \mathrm{mg}$ de triancinolona pode levar à supressão completa do eixo hipotálamo-hipófise-adrenal com duração de até oito meses por conta da dificuldade de depuração.

Dentre os efeitos mais bem destacados relacionados a triancinolona acetonida, pode-se observar manifestações clínicas sutis como mudança na distribuição e ganho de peso, erupções cutâneas, face arredondada e engrossamento de pelos da face, dentre os relatos, esses sintomas começaram a aparecer durante as 3 primeiras semanas. Ao longo do tempo, manifestações mais severas surgiram como hipertensão, Diabetes Mellitus, síndrome metabólica e necrose avascular resultando em significativa morbidade. Vale ressaltar que o diagnóstico pode ser complicado pelo fato de que a apresentação inicial da síndrome de Cushing compartilha semelhanças com a lipodistrofia associada à terapia antirretroviral, o que corrobora com o aparecimento de efeitos mais graves. (Sadarangani et al, 2014; Levine et al, 2011; Schwarze-zander et al, 2013; Alidoost et al, 2020)

Outras apresentações severas incluem miopatia induzida por esteróide e o aparecimento de infecções oportunistas devido à imunodeficiência secundária e, em alguns pacientes, a necrose avascular da cabeça femoral também foi relatada. A apresentação da insuficiência adrenal secundária relativa pode ser variável com fadiga, tontura, hipotensão e, apesar de ser associada nestes casos à SC iatrogênica, cabe ressaltar que a própria fisiopatologia da infecção pelo HIV pode favorecer a insuficiência adrenal independente do corticóide, seja pelo efeito citopático do retrovírus, seja pelo uso de antirretrovirais e/ou infecções oportunistas e neoplasias.(Sadarangani et al, 2014; Levine et al, 2011; Schwarze-zander et al, 2013; Duch et al, 1998)

No caso do uso de esteróides, o uso concomitante de ritonavir e atazanavir resulta em um catabolismo prolongado da triancinolona acetonida e subsequente aumento da concentração sérica de glicocorticóides. Essa interação forte entre a triancinolona e o ritonavir culminou, na maioria dos relatos, em insuficiência adrenal transitória com remissão dos sintomas após descontinuação de hidrocortisona (John G et al, 2013). Dessa forma, a substituição temporária após a eliminação da triancinolona por hidrocortisona em uma dose fisiológica baixa de 10-20 mg por dia pode prevenir o aparecimento de déficit de cortisol (Schwarze-zander et al, 2013). Ainda, para algumas diretrizes preconizam que deve-se considerar a dose reduzida de metilprednisolona (20-40 mg). Pode-se também haver necessidade de alterar seu regime antirretroviral para reduzir os níveis séricos de triancinolona nos casos de falência adrenal (Maviki, et al, 2013).

Destarte, a extensão do metabolismo dos corticosteróides pela isoenzima CYP3A4 é o fator principal crítico para determinar o grau de acumulação dos esteróides no organismo. Outras propriedades importantes que podem minimizar essa interação e impedir o aparecimento de síndrome de cushing são menor afinidade de ligação do fármaco ao receptor relativa de glucocorticosteróides, menor biodisponibilidade oral sistêmica, maior ligação às proteínas plasmáticas, meia-vida de eliminação mais curta e menor lipofilicidade, que se traduz em menor distribuição e ligação da droga aos tecidos (Saberi et al, 2013). Ademais, outra opção que minimizaria os danos seria preferência terapêutica por metilprednisolona, que apesar dos poucos estudos, demonstrou altos graus de evidência de menor interação. (Schwarze-zander et al, 2013)

\section{Considerações Finais}

Dessa forma, nota-se que o uso de glicocorticóides exógenos em pacientes que fazem uso de terapia antirretroviral do HIV pode induzir quadros clínicos da síndrome de Cushing. Dentre os corticoides, destacam-se a Triancinolona, Fluticasona e a Budesonida, que interagem com o ritonavir. Além disso, percebe-se que embora o uso concomitante de alguns desses fármacos com o Ritonavir não seja recomendado, a não ser que os benefícios superem os riscos, ainda são significativos os relatos de caso a respeito da Síndrome de Cushing como principal efeito adverso dessas interações. Nesse sentido, ressalta-se a 
necessidade do reconhecimento, por profissionais das diversas áreas da medicina, de fatores de risco para o desenvolvimento da SC em pacientes portadores do HIV, a partir do recordatório dos fármacos utilizados pelo paciente e a troca ou ajuste de acordo com as interações medicamentosas, levando-se em consideração também a faixa etária desses pacientes. Dessa maneira, ao identificar prováveis impactos da interação farmacológica, a partir da observação do paciente como um todo, é possível evitar a potencialização do sofrimento em pacientes já sobrecarregados pelo estigma social e pelo abalo fisiológico ocasionados pelo HIV.

\section{Referências}

Albert, N. E., Kazi, S., Santoro, J., \& Dougherty, R. (2012). Ritonavir and epidural triamcinolone as a cause of iatrogenic Cushing's syndrome. The American journal of the medical sciences, 344(1), 72-74.

Alidoost, M., Conte, G. A., Agarwal, K., Carson, M. P., Lann, D., \& Marchesani, D. (2020). Iatrogenic Cushing's syndrome following intra-articular triamcinolone injection in an HIV-infected patient on cobicistat presenting as a pulmonary embolism: case report and literature review. International Medical Case Reports Journal, 13, 229.

Arce, S. C. B., \& Aveiro, A. (2018). Complicaciones del uso prolongado de corticoides: Cushing iatrogénico. Revista Virtual de la Sociedad Paraguaya de Medicina Interna, 30-37.

Azevedo, L., Pêgo, H., Moura, T. S., \& Germano, I. (2015). Iatrogenic Cushing's syndrome and osteoporosis due to an interaction between fluticasone and ritonavir. Case Reports, 2015, bcr2015211080.

Blondin, M. C., Beauregard, H., \& Serri, O. (2013). Iatrogenic Cushing syndrome in patients receiving inhaled budesonide and itraconazole or ritonavir: two cases and literature review. Endocrine Practice, 19(6), e138-e141.

Colpitts, L., Murray, T. B., Tahhan, S. G., \& Boggs, J. P. (2017). Iatrogenic cushing syndrome in a 47-year-old HIV-positive woman on ritonavir and inhaled budesonide. Journal of the International Association of Providers of AIDS Care (JIAPAC), 16(6), 531-534.

de Araújo, L. F., Teva, I., \& de la Paz Bermúdez, M. (2014). Psychological and socio-demographic variables associated with sexual risk behavior for sexually transmitted infections/HIV. International Journal of Clinical and Health Psychology, 14(2), 120-127.

Dubrocq, G., Estrada, A., Kelly, S., \& Rakhmanina, N. (2017). Acute development of Cushing syndrome in an HIV-infected child on atazanavir/ritonavir based antiretroviral therapy. Endocrinology, diabetes \& metabolism case reports, 2017(1).

Epperla, N., \& McKiernan, F. (2015). Iatrogenic Cushing syndrome and adrenal insufficiency during concomitant therapy with ritonavir and fluticasone. Springerplus, 4(1), 1-7.

Figueiredo, J. M. N. (2017). Síndrome de Cushing iatrogénica por interacção entre Fluticasona inalada e Ritonavir (Doctoral dissertation).

Fonseca, L. K. D. S., Santos, J. V. D. O., Araújo, L. F. D., \& Sampaio, A. V. F. C. (2020). Análise da estigmatização no contexto do HIV/AIDS: Concepções de Pessoas que Vivem com HIV/AIDS. Gerais: Revista Interinstitucional de Psicologia, 13(2), 1-15.

Grierson, M. J., \& Harrast, M. A. (2012). Iatrogenic Cushing syndrome after epidural steroid injections for lumbar radiculopathy in an HIV-infected patient treated with ritonavir: a case report highlighting drug interactions for spine interventionalists. $P M \& R, 4(3), 234-237$.

Hall, J. J., Hughes, C. A., Foisy, M. M., Houston, S., \& Shafran, S. (2013). Iatrogenic Cushing syndrome after intra-articular triamcinolone in a patient receiving ritonavir-boosted darunavir. International journal of STD \& AIDS, 24(9), 748-752.

Jakeman, B., Conklin, J., Bouchonville, M., \& Thornton, K. (2015). Iatrogenic Cushing's syndrome after triamcinolone plus ritonavir-boosted atazanavir. Journal of the American Pharmacists Association, 55(2), 193-197.

John, G. R., Ollo, D., Meyer, P., Samer, C. F., \& Calmy, A. (2013). Pulmonary embolism and iatrogenic Cushing's syndrome after co-administration of injected-triamcinolone and ritonavir. Aids, 27(17), 2827-2828.

John, G. R., Ollo, D., Meyer, P., Herold, M., Samer, C. F., \& Calmy, A. (2013). Clinical course and management of iatrogenic Cushing's syndrome after coadministration of injected-triamcinolone and Ritonavir: a systematic review. Journal of Antivirals and Antiretrovirals, 5(07).

Kant, R., Cromer, M. D., Chandra, R., Munir, K., \& Verma, V. (2020). Avascular Necrosis of Both Hips From Iatrogenic Cushing's Syndrome due to Coadministration of Fluticasone and Ritonavir in an HIV-Infected Patient. Cureus, 12(8).

Levine, D., Ananthakrishnan, S., \& Garg, A. (2011). Iatrogenic Cushing syndrome after a single intramuscular corticosteroid injection and concomitant protease inhibitor therapy. Journal of the American Academy of Dermatology, 65(4), 877-878.

Mahlab-Guri, K., Asher, I., Gradstein, S., Zung, A., Radian-Sade, S., Elbirt, D., \& Sthoeger, Z. (2011). Inhaled fluticasone causes iatrogenic cushing's syndrome in patients treated with Ritonavir. Journal of Asthma, 48(8), 860-863.

Mathias, P. M., \& Epstein, E. J. (2020). Iatrogenic Cushing Syndrome In An Hiv-Infected Patient Secondary to Concomitant Therapy With Genvoya and Epidural Triamcinolone. AACE Clinical Case Reports, 6(5), e217-e220. 
Research, Society and Development, v. 10, n. 17, e34101723547, 2021

(CC BY 4.0) | ISSN 2525-3409 | DOI: http://dx.doi.org/10.33448/rsd-v10i17.23547

Maviki, M., Cowley, P., \& Marmery, H. (2013). Injecting epidural and intra-articular triamcinolone in HIV-positive patients on ritonavir: beware of iatrogenic Cushing's syndrome. Skeletal radiology, 42(2), 313-315.

Neto, H. J. C., Rosário, C. S., \& Rosário, N. A. (2010). Corticosteroides intranasais. Rev. bras. alerg. imunopatol, 33, 51-57.

Perry, M. E., Almaani, N., Desai, N., Larbalestier, N., Fox, J., \& Chilton, D. (2013). Raltegravir-induced drug reaction with eosinophilia and systemic symptoms (DRESS) syndrome-implications for clinical practice and patient safety. International journal of STD \& AIDS, 24(8), 639-642.

Répele, C. A., Spadaro, F., Reis, M. A. D., Rodrigues, D. B. R., Ferraz, M. L. D. F., \& Teixeira, V. D. P. A. (1998). Alterações morfológicas da glândula supra-renal na síndrome da imunodeficiência adquirida. Revista da Sociedade Brasileira de Medicina Tropical, 31, 257-261.

Saberi, P., Phengrasamy, T., \& Nguyen, D. P. (2013). Inhaled corticosteroid use in HIV-positive individuals taking protease inhibitors: a review of pharmacokinetics, case reports and clinical management. HIV medicine, 14(9), 519-529.

Sadarangani, S., Berg, M. L., Mauck, W., \& Rizza, S. (2014). Iatrogenic cushing syndrome secondary to ritonavir-epidural triamcinolone interaction: an illustrative case and review. Interdisciplinary perspectives on infectious diseases, 2014.

Schwarze-Zander, C., Klingmüller, D., Klümper, J., Strassburg, C. P., \& Rockstroh, J. K. (2013). Triamcinolone and ritonavir leading to drug-induced Cushing syndrome and adrenal suppression: description of a new case and review of the literature. Infection, 41(6), 1183-1187.

Tashkin, D. P., Lipworth, B., \& Brattsand, R. (2019). Benefit: risk profile of budesonide in obstructive airways disease. Drugs, 79(16), 1757-1775.

Tiruneh, F., Awan, A., Didana, A., \& Doshi, S. (2017). Preventing Cushing: iatrogenic Cushing syndrome due to ritonavir-fluticasone interaction. Cureus, 9(7).

Van Den Berg, S. A. A., van`t Veer, N. E., Emmen, J. M. A., \& Van Beek, R. H. T. (2017). Fluticasone furoate induced iatrogenic Cushing syndrome in a pediatric patient receiving anti-retroviral therapy. Endocrinology, diabetes \& metabolism case reports, 2017(1).

Yoganathan, K., David, L., Williams, C., \& Jones, K. (2012). Cushing's syndrome with adrenal suppression induced by inhaled budesonide due to a ritonavir drug interaction in a woman with HIV infection. International journal of STD \& AIDS, 23(7), 520-521. 